\title{
Efficacy of birinapant in combination with carboplatin in targeting platinum-resistant epithelial ovarian cancers
}

\author{
TANYA SINGH $^{1,2}$, ADAM NEAL ${ }^{1,2}$, GABRIELLA DIBERNARDO $^{1,2}$, NEELA RAHESEPARIAN $^{1,2}$, \\ NEDA A. MOATAMED ${ }^{3}$ and SANAZ MEMARZADEH ${ }^{1,2,4-6}$
}

\author{
${ }^{1}$ Department of Obstetrics and Gynecology, David Geffen School of Medicine, University of California Los Angeles; \\ ${ }^{2}$ UCLA Eli and Edythe Broad Center of Regenerative Medicine and Stem Cell Research, \\ University of California Los Angeles; ${ }^{3}$ Department of Pathology and Laboratory Medicine, David Geffen School of Medicine, \\ University of California Los Angeles; ${ }^{4}$ Molecular Biology Institute, University of California Los Angeles; \\ ${ }^{5}$ UCLA Jonsson Comprehensive Cancer Center, University of California Los Angeles, Los Angeles, CA 90095; \\ ${ }^{6}$ The VA Greater Los Angeles Healthcare System, Los Angeles, CA 90073, USA
}

Received October 14, 2021; Accepted November 25, 2021

DOI: $10.3892 /$ ijo.2022.5325

\begin{abstract}
Patients diagnosed with epithelial ovarian cancers (EOCs) often suffer from disease relapse associated with the emergence of resistance to standard platinum-based chemotherapy. Treatment of patients with chemo-resistant disease remains a clinical challenge. One mechanism of chemoresistance includes overexpression of pro-survival proteins called inhibitors of apoptosis (IAP) which enable cancer cells to evade apoptosis. Due to their anti-apoptotic activity, association with poor prognosis, and correlation with therapy resistance in multiple malignancies, IAP proteins have become an attractive target for development of anticancer therapeutics. Second mitochondrial activator of caspase (SMAC) mimetics are the most widely used IAP antagonists currently being tested in clinical trials as a monotherapy and in combination with different chemotherapeutic drugs to target different types of cancer. In the present study, the antitumor efficacy of combination therapy with birinapant, a bivalent SMAC mimetic compound, and carboplatin to target platinum-resistant EOC cells was investigated. A 3D organoid bioassay was utilized to test the efficacy of the combination therapy in a panel of 7 EOC cell lines and 10 platinum-resistant primary patient tumor samples. Findings from the in vitro studies demonstrated that the birinapant and carboplatin combination was
\end{abstract}

Correspondence to: Dr Sanaz Memarzadeh or Dr Tanya Singh, Department of Obstetrics and Gynecology, David Geffen School of Medicine, University of California Los Angeles, 610 Charles E. Young Drive East, 3018 Terasaki Life Sciences Building, Los Angeles, CA 90095, USA

E-mail: smemarzadeh@mednet.ucla.edu

E-mail: tanyasingh@mednet.ucla.edu

Key words: ovarian cancer, platinum resistance, IAP, SMAC mimetics, birinapant, carboplatin, drug synergy, organoids effective in targeting a subset of ovarian cancer cell lines and platinum-resistant primary patient tumor samples. This combination therapy was also effective in vitro and in vivo in targeting a platinum-resistant patient-derived xenograft (PDX) model established from one of the patient tumors tested. Overall, our study demonstrated that birinapant and carboplatin combination could target a subset of platinum-resistant ovarian cancers and also highlights the potential of the 3D organoid bioassay as a preclinical tool to assess the response to chemotherapy or targeted therapies in ovarian cancer.

\section{Introduction}

Ovarian cancer (OC) is a deadly malignancy of the female reproductive system responsible for a high number of deaths each year. It is estimated that by the end of 2021 approximately 21,410 will be diagnosed with ovarian cancer, while 13,770 women will die from this disease in the US alone (1). Epithelial ovarian cancers (EOCs) are the most common subtype of ovarian tumor and may arise from epithelial cells lining the ovaries, peritoneum and fallopian tubes (2). Frontline treatment for EOCs involve tumor debulking surgery followed by platinum-based chemotherapy (3). Patients with a large tumor burden may also undergo neoadjuvant chemotherapy treatment in an effort to reduce the tumor volume prior to surgery (3). After frontline treatment, patients with advanced $\mathrm{OC}$ often receive maintenance therapy to prolong their progression-free survival (PFS) (4). The National Comprehensive Cancer Network (NCCN) guidelines recommend several maintenance therapies, including anti-angiogenic bevacizumab and poly(ADP-ribose) polymerase (PARP) inhibitors for patients with advanced disease (4).

Despite these therapeutic advancements, most patients diagnosed with metastatic OC experience disease relapse. Repeated tumor recurrences result in the emergence of chemotherapy resistance, particularly to platinum-based drugs, and patients eventually succumb to platinum-resistant disease (5). Effective treatment of patients with chemo-resistant disease 
remains a major clinical challenge and is the focus of the present study.

Response to platinum-based chemotherapy is dependent on multiple parameters including tumor biology and histology, molecular alterations, and tumor microenvironment $(6,7)$. Patient sensitivity to platinum drugs is determined based on the progression-free interval after completing multiple cycles of chemotherapy infusion. Patients are deemed platinum-sensitive if disease relapse occurs at more than 6 months after administration of chemotherapy while those with tumor recurrence less than 6 months after chemotherapy are classified as platinum-resistant (6). For treatment of platinum-resistant patients, NCCN guidelines recommend use of single nonplatinum-based chemotherapy drugs such as docetaxel, etoposide, gemcitabine, liposomal doxorubicin, and topotecan (4). These guidelines also recommend enrollment of patients with platinum-resistant $\mathrm{OC}$ in clinical trials testing new therapies. Overall, treatment of chemoresistant disease is focused on relieving patients of disease-related symptoms, controlling adverse reactions to drugs, and maintaining quality of life. Hence, there is a critical need for novel therapeutic interventions that can effectively reduce the mortality rate and prolong the overall survival of patients with platinum-resistant OC.

Several molecular factors may contribute to the development of therapy resistance in OC such as reduced cellular accumulation of platinum due to altered expression of membrane transporter proteins, increased drug efflux, upregulated DNA repair, reduced apoptosis, and increased autophagy (8). In the present study, we exploit defects in apoptotic processes as a possible therapeutic target for treating platinum-resistant $\mathrm{OC}$. Cancer cells are known to evade apoptosis by overexpression of a highly conserved class of anti-apoptotic proteins called inhibitor of apoptosis (IAP) proteins (9). IAP proteins exert their anti-apoptotic activity either through direct inhibition of caspases or through indirect ubiquitination of caspase proteins (9). Eight IAP proteins have been identified in humans [reviewed in Finlay et al (10)]. Out of these IAP proteins, some of the most extensively studied proteins include cellular IAP proteins (cIAP1 and cIAP2) and XIAP (9). Deregulated cIAP and XIAP levels have been correlated with response to therapy or disease progression in different types of cancer including ovarian cancer (11-14). Survivin, another important member of the IAP family has also been investigated in different cancer types and has been linked to tumor progression and metastasis $(15,16)$, therapy-resistance $(17,18)$ and poor prognosis $(19,20)$.

Second mitochondrial activator of caspase (SMAC) mimetics are small molecules designed to mimic endogenous SMAC proteins released by mitochondria (21). SMAC mimetic molecules predispose cells to apoptosis by inhibiting IAP proteins (21). Multiple clinical trials are exploring the potential of such IAP inhibitors administered as a single agent and in combination with chemotherapeutics for treatment of various types of cancer [reviewed in Morrish et al (21)]. We hypothesize that combining SMAC mimetics with carboplatin, the standard chemotherapy drug used in the treatment of OC, may enhance carboplatin-induced cell death. To test this hypothesis, we have utilized birinapant, a highly potent SMAC mimetic known to target cIAP1/2 and XIAP in combination with carboplatin to target platinum-resistant ovarian cancer cells. Birinapant is a promising IAP protein antagonist that has demonstrated antitumor activity in preclinical models of head and neck squamous cell carcinoma (HNSCC) (22), and other solid tumors including triple-negative breast cancer $(23,24)$, non-small cell lung cancer (25), and ovarian cancer (26). The outcome of a clinical trial testing birinapant as a monotherapy in advanced ovarian, fallopian tube, and peritoneal cancers (NCT01681368) has demonstrated its tolerability in a dose-dependent manner but was not found efficacious as a single agent (27).

In the present study, the efficacy of birinapant and carboplatin combination therapy was evaluated using in vitro and in vivo models of platinum-resistant EOCs. A 3D organoid bioassay was utilized to test the in vitro therapeutic efficacy of co-therapy across a panel of ovarian cancer cell lines and platinum-resistant primary patient tumor samples. The results demonstrated that birinapant with carboplatin was effective in targeting a subset of ovarian cancer cell lines and platinum-resistant patient ovarian tumors.

\section{Materials and methods}

Cell lines and primary patient tumor samples. Ovarian cancer cell lines including SKOV3, OVCAR3, CaOV3, Kuramochi and OAW28 were purchased from the American Type Culture Collection (ATCC). OVCAR4 and OVCAR8 cell lines were obtained from the National Cancer Institute Division of Cancer Treatment and Diagnosis (NCI/DCTD) repository through Material Transfer Agreement (MTA). Cell lines were grown in recommended media (RPMI supplemented with $10 \%$ FBS or DMEM supplemented with $10 \%$ FBS) in a $5 \% \mathrm{CO}_{2}$ humidified incubator at $37^{\circ} \mathrm{C}$ and used within 10 passages. All cell lines used in this study were frequently authenticated by STR analysis. Primary patient tumor samples were obtained from patients after providing informed consent through protocols approved by the UCLA Office of the Human Research Protection Program (IRB\# 10-000727). Patients ages ranged from 43 to 75 years. Tumor samples were mechanically and enzymatically dissociated with $1 \mathrm{mg} / \mathrm{ml}$ collagenase and dispase solution (Gibco; Thermo Fisher Scientific, Inc.) and cryopreserved in buffer (90\% FBS, 10\% DMSO) for experimental use.

In vitro 3 D organoid bioassay. As previously described $(28,29)$, in this bioassay, 5,000 cells/well were suspended in Matrigel matrix (Corning Inc.) and MammoCult growth medium (STEMCELL Technologies) and plated around the rim of the wells of a 96-well plate. Organoids were allowed to grow for 1-2 days followed by drug treatment in a dose-dependent manner for 3 consecutive days. After drug treatment, cell viability was determined using an ATP luminescence assay (CellTiter-Glo 3D viability Assay kit; Promega Corp.). For testing platinum sensitivity, each cell line was treated with carboplatin at increasing concentrations of $0,10,25,50,100$, 150,200 and $250 \mu \mathrm{M}$. For co-therapy testing, cells were treated with a range of doses of carboplatin $(0-50 \mu \mathrm{M})$ and birinapant (0-50 nM).

As a measure for quality control, cryopreserved primary tumor samples with cell viability less than $60 \%$ after 
thawing were excluded from the study. Only those primary samples that formed visible organoids in the 3D organoid bioassay were included in this study, confirmed by examination under a microscope. Assay results were rejected if the luminescence value in vehicle-treated wells were found to be similar to the blank control [Matrigel and growth media]. Staurosporine-treated cells were used as a positive control for cell death in each experiment.

Drug synergy analysis. Synergy between birinapant and carboplatin was quantified using the Loewe additivity index model available in a web-based package SynergyFinder 2.0 (30). Synergy scores were calculated across all tested drug concentrations and combinations and visualized as a two-dimensional interaction surface over the dose matrix (Loewe synergy plots). Based on the Loewe additivity model, a summary synergy score was generated. Drug interactions were classified as likely synergistic (score $>10$ ), antagonistic (score $<-10$ ), or additive (score -10 to +10 ). Synergy scores shown in this study were obtained from the SynergyFinder 2.0 tool as of 10/04/2021.

TCGA data analysis. Information regarding the genetic alterations in $c I A P 1, c I A P 2$ and XIAP genes were obtained from the Cancer Genome Atlas (TCGA) database through cBioPortal (http://www.cbioportal.org). Data shown in this study were extracted from PanCancer datasets as of 10/04/2021. We focused on TCGA data for 8 cancer types known to be treated with platinum-based chemotherapy (31-34).

Western blot analysis. For assessing the endogenous levels of IAP proteins in 7 EOC cell lines, growing cells were harvested, lysed using RIPA lysis buffer supplemented with protease inhibitor cocktail (Thermo Fisher Scientific, Inc.). Cell lysates were centrifuged at $12,000 \mathrm{x} \mathrm{g}$ for $10 \mathrm{~min}$ at $4^{\circ} \mathrm{C}$. The supernatant was collected, and protein concentration was measured using a BCA protein assay kit (Thermo Fisher Scientific, Inc.). Equal amounts $(40 \mu \mathrm{g})$ of protein were loaded in each lane and resolved on NuPAGE 4-12\% Bis-Tris gels (Thermo Fisher Scientific, Inc.). Resolved proteins were transferred to nitrocellulose membranes (Millipore), probed with appropriate antibodies and detected by chemiluminescent reagent (Millipore, WBKLS0500). Protein bands were visualized using a Bio-Rad ChemiDoc Imaging system (Bio-Rad Laboratories).

For measuring the IAP protein levels in SKOV3 and OVCAR8 cells after co-therapy treatment, both cell lines were treated in 2D cell culture with carboplatin alone, birinapant alone, or the combination of the two drugs at a concentration corresponding to the $48 \mathrm{~h}$ half maximal inhibitory concentration $\left(\mathrm{IC}_{50}\right)$ of each drug. After $24 \mathrm{~h}$ of drug treatment, cells were lysed and processed the same way as mentioned above. For measuring IAP protein levels in PDX subcuticular tumors, an additional step of sonication (Thermo Fisher Scientific sonicator) was performed after tumors were lysed in RIPA lysis buffer.

Antibodies. Antibodies used in this study included: cIAP1 [Cell Signaling Technology, Inc. (CST), cat. no. 7065], cIAP2 (CST, cat. no. 3130), XIAP (CST, cat. no. 14334), GAPDH (CST, cat. no. 5174), Ki67 (Agilent Technologies, cat. no. M724001-2), and Pax8 (Proteintech, cat. no. 10336-1-AP). For western blot experiments, cIAP1, cIAP2, XIAP and GAPDH antibodies were used at a dilution of 1:1,000. For IHC experiments Ki67 and Pax8 antibodies were used at a dilution of 1:100 and 1:500 respectively.

Apoptosis analysis by flow cytometry. Apoptosis was detected by Annexin V and PI staining using BD Pharmingen Apoptosis Detection Kit (cat. no. 556547). Cells (0.5 million/well) were seeded in 6-well plates. Cells were treated with various concentration of carboplatin, birinapant, or the co-therapy for 72 h. Cells were then harvested and stained with Annexin V/PI following the manufacturer's protocol. Cell death was analyzed using a BD FACS ARIA flow cytometer and FlowJo software (version 10.8.0; BD Biosciences). Cell death was represented as the percentage of Annexin $\mathrm{V}^{+}$cell population after drug treatment. For each cell line, three independent experiments were performed.

Tumor necrosis factor (TNF) $\alpha$ ELISA. Cells were seeded at a density of 0.5 million cells/well in a 6-well plate and allowed to grow until approximately $80 \%$ confluency was reached. Cells were then washed with 1X PBS, incubated with fresh media, and treated with carboplatin, birinapant, or co-therapy at a concentration corresponding to the $48 \mathrm{~h} \mathrm{IC}_{50}$ of each drug. After $72 \mathrm{~h}$ of drug treatment, $100 \mu \mathrm{l}$ of culture supernatant was collected from each treatment group for TNF $\alpha$ measurement by ELISA kit (Thermo Fisher Scientific, Inc., cat. no. BMS223HS). Recombinant TNF $\alpha$ protein was used as a positive control in these experiments. Two independent experiments were performed with duplicate wells for each condition.

Neutralization of TNF $\alpha$ molecules. Cells were seeded at a density of 5,000 cells/well in 96-well plate. Cells were then pre-treated with $10 \mu \mathrm{g} / \mathrm{ml}$ of anti-TNF $\alpha$ antibody (R\&D Systems, cat. no. MAB610-100) for $2 \mathrm{~h}$ followed by addition of carboplatin, birinapant, or combination of two drugs at the $48 \mathrm{~h} \mathrm{IC}_{50}$ of each drug. After $72 \mathrm{~h}$ of drug treatment, cell viability was measured using an ATP luminescence assay (CellTiter-Glo 3D viability Assay kit, Promega Corp.). IgG antibody was used as a non-specific antibody control. Two independent experiments were performed with triplicate wells for each condition in each cell line tested.

Establishment of a platinum-resistant patient-derived xenograft model.PDX models were established from a primary pleural effusion sample, EOC2, clinically classified as recurrent, platinum-resistant high-grade serous ovarian cancer. For PDX generation, 1 million cryopreserved cells were injected as a cell suspension in the intraperitoneal space of a female NSG mouse. The cells were re-passaged in mice twice. At the end of the second passage, PDX cells were harvested and evaluated by histological and genomic analyses. Short tandem repeat (STR) analyses performed using extracted genomic DNA from PDX cells demonstrated that this PDX retained greater than $90 \%$ identity to its parental tumor. Single nucleotide polymorphism analysis was also performed, confirming relatedness of PDX cells and parental tumor cells. 
Immunohistochemistry (IHC) staining. Histologic slides containing PDX subcuticular tumor fragments from each cohort after drug treatment were reviewed by a gynecology pathologist NAM. PAX8 immunostaining was evaluated for nuclear expression to confirm Mullerian origin of the tumors. Ki67 was also evaluated for nuclear staining for proliferation index. Scoring was based on the estimation of the percentage of tumor cells stained by the antibody visualized by light microscopy. The mitotic figures were counted on hematoxylin and eosin stained slides on 10 high power fields (40x objective). Mean number of mitosis between the treatment groups was compared by ANOVA with non-constant variance allowed.

Animals. All animal experiments were approved by the UCLA Animal Research Committee (protocol 2008-153) and conducted under the supervision of the UCLA Division of Laboratory Animal Medicine. Six- to eight-week-old female NSG mice (NOD.Cg-Prkd $d^{s c i d} / / 2 \mathrm{rg}^{\text {tml } w_{j l} l} / \mathrm{Sz} J$ ) were purchased from Jackson Laboratories for all in vivo experiments. All mice were housed in specific pathogen-free (SPF) facilities. They were kept in autoclaved cages with sterile bedding and food. Mice were anesthetized by isoflurane gas in oxygen. Following the Institutional Animal Care and Use Committee (IACUC) guidelines, 3-5\% isoflurane was used for initial induction and $1-3 \%$ was used for maintenance. For euthanization, mice were exposed to gradually increasing concentrations of $\mathrm{CO}_{2}$ at a flow rate ranging between 30-70\% chamber volume/min as per the American Veterinary Medical Association (AVMA) guidelines. Cervical dislocation was used as a confirmatory method of euthanasia after $\mathrm{CO}_{2}$ exposure. At the time of euthanization, tumor volume of each mouse was $<1,000 \mathrm{~mm}^{3}$ consistent with (IACUC) guidelines.

Statistical methods. Results are reported as means \pm standard error. For normally distributed data, the P-values for comparing means were calculated using one-way analysis of variance (ANOVA), two-way ANOVA or two way (mixed) repeated measure ANOVA (tumor mean comparison) when animals were measured repeatedly over time. When data were not normal as indicated by the Shapiro-Wilks test, P-values were computed with non-parametric Kruskal-Wallis test. Calculations were performed using $\mathrm{R}$ version 4.0.5 (R Foundation for Statistical Computing, Vienna, Austria).

Tumor volumes were calculated using the modified ellipsoid formula: $1 / 2 \times$ length $\mathrm{x}$ (width) ${ }^{2}$. The baseline volume on day 0 (start of drug treatment) was subtracted from all subsequent tumor volumes of the same animal since day 0 baseline was not the same for all animals. After this standardization, the relation between tumor volume and time (day) was approximately linear in each animal. Thus, the growth rate in $\mathrm{mm}^{3}$ per day for each animal was computed via linear regression on each animal and the mean rates were compared among the four groups using a one-way ANOVA where the variances were allowed to be heterogeneous.

\section{Results}

Utilization of a $3 D$ in vitro bioassay for testing the platinum sensitivity of human ovarian cancer cell lines. One of the challenges in the treatment of $\mathrm{OC}$ is the absence of biomarkers that could prospectively predict patients' response to standard platinum-based chemotherapy. OC tumors are characterized as platinum-sensitive or platinum-resistant only after chemotherapy drugs are administered. Hence, in vitro drug response assays that are designed to test and predict the sensitivity of patient tumor cells to existing chemotherapies may provide a useful tool for treatment of OC patients. Although these assays hold great promise in the field of precision medicine, they have not yet proven efficacious for clinical application and therefore, no such assay has been FDA approved for clinical use at this time (35). In the present study, a 3D organoid-based drug testing platform (28) was utilized to assess the response of OC cell lines to carboplatin. Platinum sensitivity of the OC cell lines measured in this bioassay were correlated with reported response to platinum drugs for each cell line $(36,37)$.

In this bioassay $(28,29)$, OC cells were grown as 3D organoids embedded within an extracellular matrix hydrogel called Matrigel (Fig. 1A). On the first day of this bioassay, a mixture of cells and Matrigel were plated around the rims of wells in a 96-well plate and overlaid with growth media. Cells were allowed to generate organoids for $24 \mathrm{~h}$. Growing organoids were treated with increasing concentrations of carboplatin $(0-250 \mu \mathrm{M})$ for three consecutive days, with daily drug replenishment. After drug treatment, the viability of the cells was measured using an ATP-based luminescence assay (CellTiter Glo, Promega Corp.). Sensitivity to carboplatin was determined using an $\mathrm{IC}_{50}$ value, defined as the drug concentration causing 50\% inhibition. $\mathrm{IC}_{50}$ values for each cell line were obtained from carboplatin dose response curves generated using the ATP-based viability assay.

The viability plots for each cell line demonstrated a dose-dependent decrease in cell viability. Differential sensitivity to carboplatin was noted in all cell lines tested (Fig. 1B). Based on the $\mathrm{IC}_{50}$ of carboplatin, OVCAR3 cells were found to be carboplatin sensitive $\left(\mathrm{IC}_{50}<40 \mu \mathrm{M}\right)$, Kuramochi and OVCAR8 cells were highly carboplatin resistant $\left(\mathrm{IC}_{50}>85 \mu \mathrm{M}\right)$. The remaining cell lines exhibited intermediate carboplatin resistance (Fig. 1C). Platinum sensitivity measured with this bioassay correlated with platinum sensitivity of each cell line reported by others $(36,37)$.

This 3D organoid bioassay was capable of classifying cell lines as carboplatin-sensitive vs. carboplatin-resistant. Since organoids are known to better recapitulate in vivo tumor architecture (38), this bioassay may provide a tool for testing single drug or combination therapies in ovarian cancer. Therefore, next this bioassay was utilized to test the efficacy of a combination therapy in targeting platinum resistant ovarian cancer cells.

A subset of human platinum-resistant ovarian cancer cell lines was targeted with a combination of carboplatin and birinapant in vitro. Cancer cells are known to overexpress highly conserved anti-apoptotic proteins called inhibitor of apoptosis (IAP) proteins (9). These proteins are predominantly known for inhibiting apoptotic cell death via regulation of caspases and are reported to be involved in tumor cell survival, chemo-resistance, disease progression, and poor prognosis [reviewed in LaCasse et al (39)]. Data from The Cancer Genome Atlas (TCGA) database also demonstrated that gene amplification is a common alteration in key IAPs, namely 

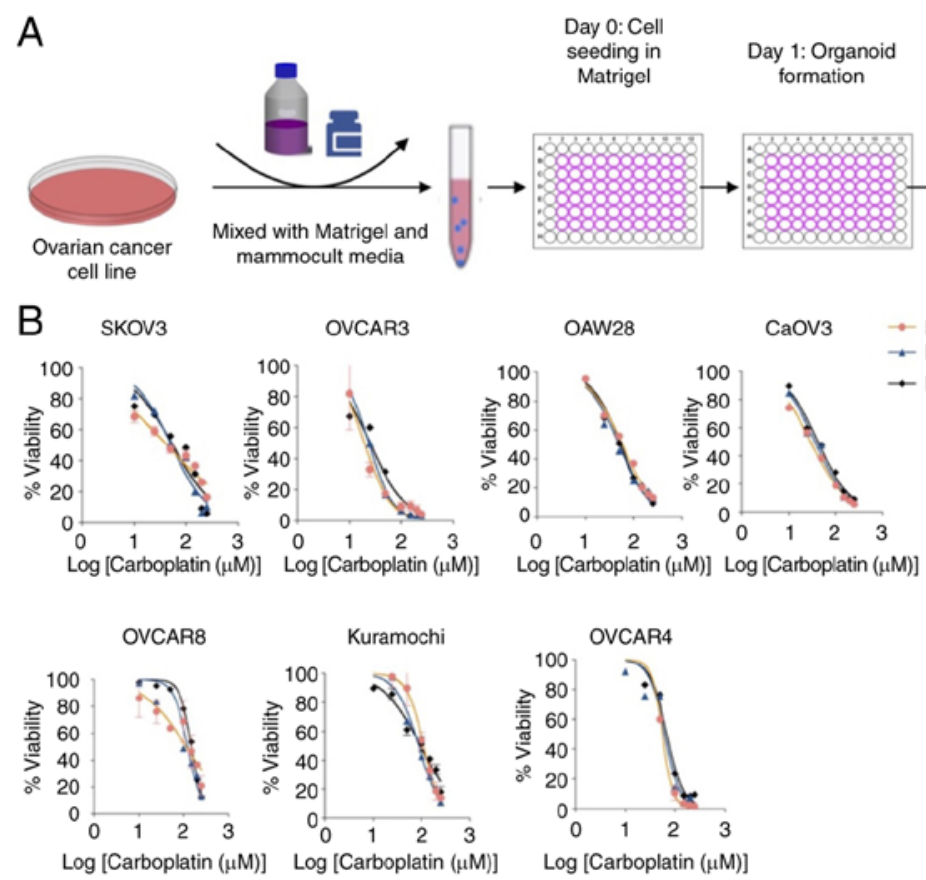

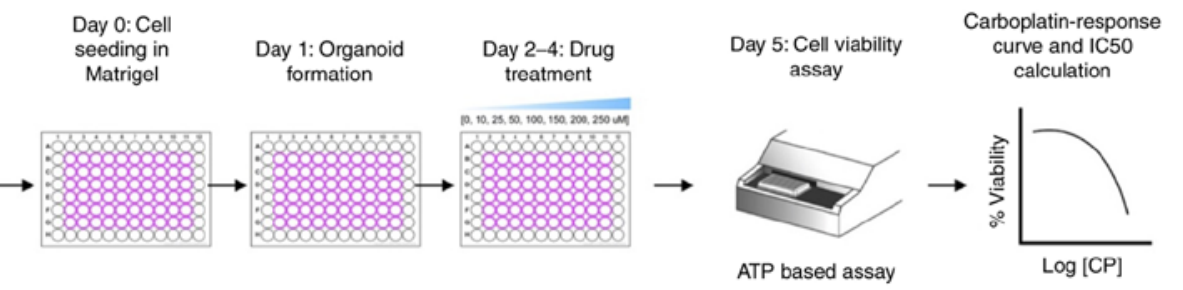

C
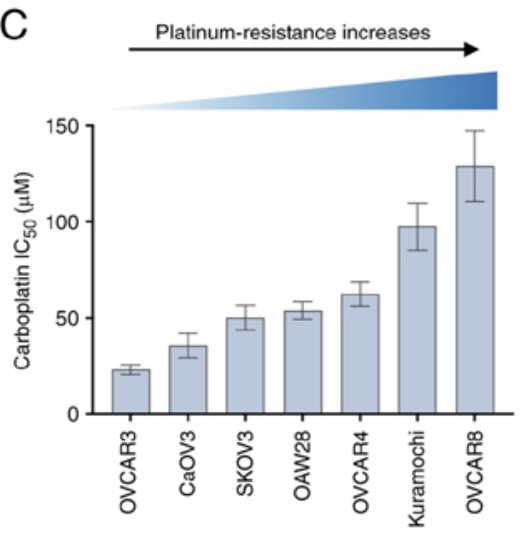

Figure 1. Carboplatin sensitivity of ovarian cancer cell lines as measured by the 3D organoid bioassay. (A) Workflow for testing platinum sensitivity of ovarian cancer cell lines in vitro. Cell line-derived organoids were treated with increasing concentrations of carboplatin (0-250 $\mu \mathrm{M})$ daily for 3 consecutive days followed by measurement of cell viability using an ATP-based luminescence assay. (B) Carboplatin dose response curves for 7 epithelial ovarian cancer cell lines. A dose-dependent decrease in cell viability was observed for each cell line. (C) The carboplatin half maximal inhibitory concentration ( $\left.\mathrm{IC}_{50}\right)$ value for each cell line was determined after $72 \mathrm{~h}$ of drug treatment. Data shown represents 3 independent experiments for each cell line.

cIAP1, cIAP2, and XIAP in ovarian cancer and other cancers treated with platinum-based chemotherapy drugs (31-34) (Fig. S1A). Taken together, this evidence provides a rationale to activate apoptosis in platinum-resistant ovarian cancer cells by targeting IAP proteins. We combined birinapant, a small molecule that mimics natural IAP protein antagonist SMAC, with carboplatin to target platinum-resistant OC cell lines in vitro. Due to its pro-apoptotic activity and tolerability, birinapant and several other SMAC mimetic molecules have been evaluated in early clinical trials as a monotherapy or in combination with other chemotherapeutic drugs to target different types of cancers [reviewed in Morrish et al (21)]. The combination of SMAC mimetics, including birinapant and carboplatin, has also been previously explored and demonstrated efficacy in targeting OC cell lines using preclinical models $(26,40)$. In this study, we expanded on these previous investigations by testing the in vitro efficacy of birinapant and carboplatin combination across a panel of 7 EOC cell lines using the 3D organoid bioassay. This 3D organoid bioassay offers several advantages. First, as a preclinical model for drug testing, it retains complex tumor architecture similar to in vivo tumors (28). Second, it is compatible with automation and high throughput drug screening allowing testing of combination therapies in a scalable and reproducible way (28).

Therapeutic target proteins of birinapant (cIAP1/2 and XIAP) were evaluated in these 7 cell lines using western blot analysis (Fig. S1B). For co-therapy testing, cell line-derived organoids were treated with increasing concentrations of carboplatin $(0-50 \mu \mathrm{m})$ and birinapant $(0-50 \mathrm{nM})$ in the 3D-organoid bioassay (Fig. 2A). After 3 days of drug treatment, cell viability was measured using an ATP-based assay (Fig. S1C). Drug synergy was scored using the Loewe additivity model available in the online package SynergyFinder 2.0 (30). Four out of the 7 cell lines (OVCAR8, SKOV3, Kuramochi, and OAW28) demonstrated a positive synergy score that indicates increased cell death upon co-treatment with birinapant and carboplatin compared to the sum of the single agents (Fig. 2B and C). The combined effects were found to be likely additive in the OVCAR4 cell line while the effects of these two drugs were found to be likely antagonistic in OVCAR3 and $\mathrm{CaOV} 3$ cell lines (Fig. $2 \mathrm{~B}$ and $\mathrm{C}$ ). Overall, these in vitro results demonstrated that addition of birinapant to carboplatin treatment enhanced cell death in a subset of OC cell lines.

\section{Combination treatment induces apoptosis in platinum-} resistant ovarian cancer cell lines. Both birinapant and carboplatin are known to induce cell death. Birinapant-mediated cell death occurs by inhibition of IAP proteins (21) while carboplatin forms DNA lesions that block DNA synthesis and results in cell death (41). Therefore, the combined effect of carboplatin and birinapant on cellular apoptosis was investigated by flow cytometry. We selected a highly platinum-resistant cell line, OVCAR8, and a moderately platinum resistant cell line, SKOV3, as these two cell lines demonstrated Loewe synergy scores greater than 10 when treated with carboplatin and birinapant combination in vitro (Fig. 2C).

Quantification of drug-induced cell death was facilitated by Annexin V/PI staining using flow cytometry. Both SKOV3 and OVCAR8 cells were treated with carboplatin, birinapant, or the combination of both drugs in $2 \mathrm{D}$ cell culture at concentrations corresponding to $\mathrm{IC}_{50}$ values of each drug. After $72 \mathrm{~h}$ of drug treatment, cells were harvested, stained with Annexin V/PI and analyzed by flow cytometry (Fig. 3A). The percentage of dead cells (Annexin $\mathrm{V}^{+}$) was significantly increased when either 


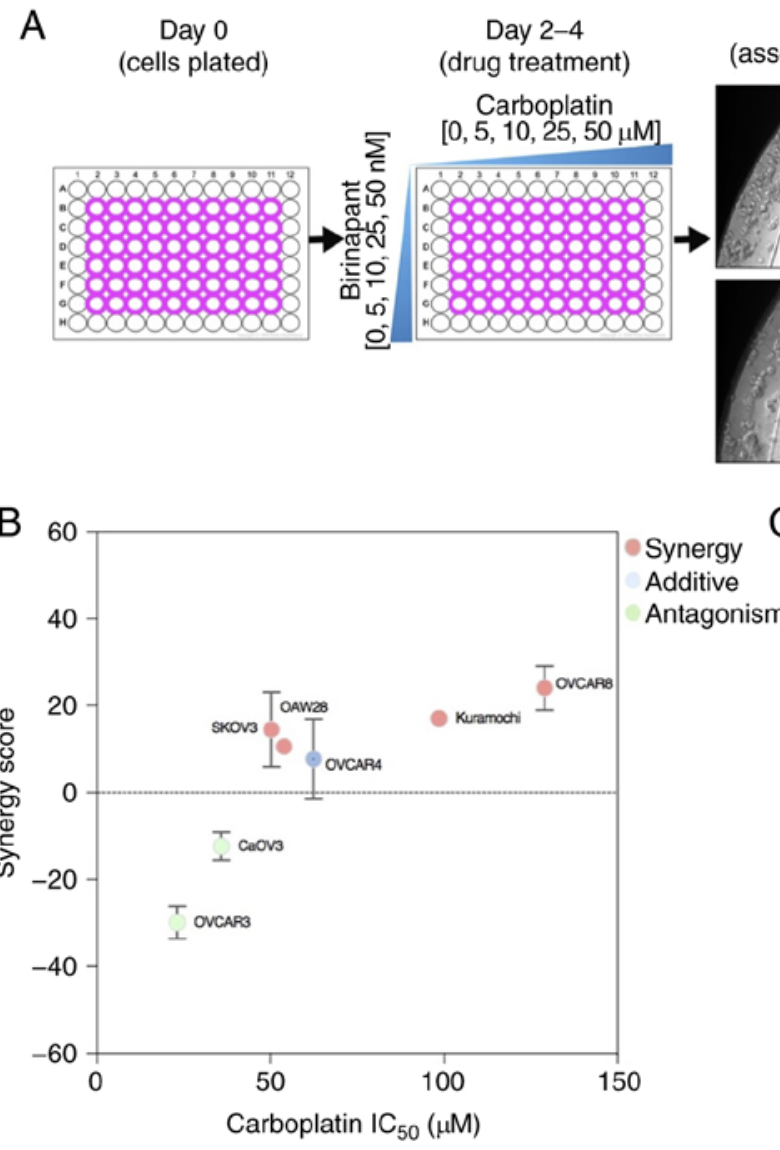

$$
\text { Day } 5
$$
(assessment of cell viability) Synergy score calculation
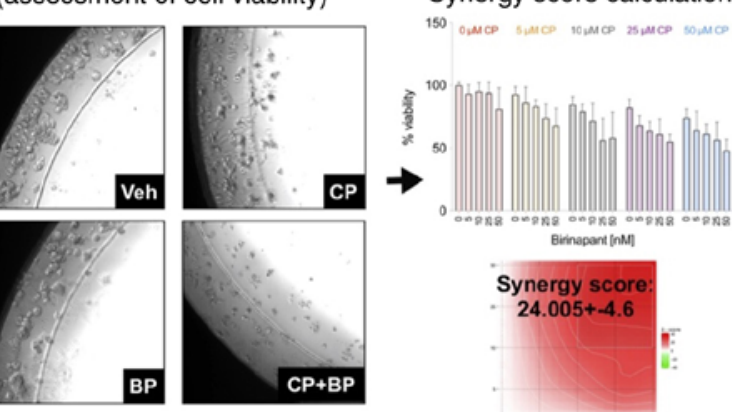

C

\begin{tabular}{|c|c|c|}
\hline Cell line & Synergy score & Type of drug interaction \\
\hline SKOV3 & $14.533 \pm 8.54$ & Synergistic \\
\hline OAW28 & $10.619 \pm 1.17$ & Synergistic \\
\hline Kuramochi & $17.081 \pm 1.83$ & Synergistic \\
\hline OVCAR8 & $24.005 \pm 5.06$ & Synergistic \\
\hline OVCAR4 & $7.73 \pm 9.19$ & Additive \\
\hline CaOV3 & $-12.343 \pm 3.25$ & Antagonistic \\
\hline OVCAR3 & $-29.878 \pm 3.77$ & Antagonistic \\
\hline
\end{tabular}

Figure 2. Testing the efficacy of carboplatin and birinapant combination in targeting epithelial ovarian cancer cell lines using the 3D organoid bioassay. (A) Experimental schema. Cell line-derived organoids were treated with increasing concentrations of birinapant $(0-50 \mathrm{nM})$ and carboplatin (0-50 $\mu \mathrm{M})$ for 3 consecutive days with daily drug replenishment followed by cell viability assessment using the ATP-based luminescence assay. Cell viability values were used to calculate drug synergy scores using the SynergyFinder tool. Data for OVCAR8 cells are shown. (B) Loewe synergy scores for each cell line plotted against its corresponding carboplatin half maximal inhibitory concentration $\left(\mathrm{IC}_{50}\right)$ value. $(\mathrm{C})$ Type of drug interaction for carboplatin and birinapant co-therapy treatment in each cell line, represented as likely synergistic (red), additive (blue), or antagonistic (green). Data represented as synergy score $\pm 95 \%$ confidence interval from 3 independent experiments for each cell line.

cell line was treated with the co-therapy $(\mathrm{CP}+\mathrm{BP})$ compared to single agents (CP or BP) (Fig. 3B and C). Degradation of cIAP1 was detected in both cell lines by birinapant alone, or in combination with carboplatin (Fig. S2). However, birinapant had no effect on XIAP expression levels in these cell lines.

Several studies have reported that SMAC mimetics including birinapant stimulate cells to release cytokine $\mathrm{TNF} \alpha$ which binds to membrane-bound TNF receptors and triggers an apoptosis-signaling pathway $(42,43)$. The role of TNF $\alpha$ in co-therapy targeted SKOV3 and OVCAR8 cells was examined by measuring the release of TNF $\alpha$ molecules using ELISA. In this experiment, both cell lines were treated with carboplatin (CP), birinapant (BP), or the combination of the two agents $(\mathrm{CP}+\mathrm{BP})$ in $2 \mathrm{D}$ cell culture at concentrations corresponding to the $\mathrm{IC}_{50}$ of each drug (Fig. 3D). After $72 \mathrm{~h}$ of drug treatment, $100 \mu \mathrm{l}$ of culture supernatant was collected from each treatment group for TNF $\alpha$ measurement by ELISA. Results demonstrated that co-therapy treated OVCAR8 and SKOV3 cells secreted significantly more $\mathrm{TNF} \alpha$ compared to carboplatin alone (Fig. 3D, right panel). This suggests that TNF $\alpha$ signaling may also contribute to overall drug synergy observed in these cell lines. To further confirm the role of TNF $\alpha$ in mediating pro-apoptotic signaling, anti-TNF $\alpha$ neutralizing antibody was used to block the interaction between TNFa and its membrane bound TNF receptor. In this experiment, both cell lines were pre-treated with anti-TNF $\alpha$ antibody for $2 \mathrm{~h}$ followed by addition of carboplatin (CP), birinapant (BP), or the combination of the two agents $(\mathrm{CP}+\mathrm{BP})$ for $72 \mathrm{~h}$ (Fig. 3E). After drug treatment, cell viability was measured using an ATP-based luminescence assay. Neutralization of TNF $\alpha$ molecules with anti-TNF $\alpha$ antibody was found to partially reverse co-therapy-induced cytotoxicity. An increase in cell viability was observed in SKOV3 and OVCAR8 cells treated with the co-therapy following pre-treatment with the anti-TNF $\alpha$ antibody (Fig. 3E, right panel).

Overall, the findings demonstrated that apoptosis was induced in the co-therapy-treated platinum-resistant OVCAR8 and SKOV3 cells. It also suggests that mechanisms of drug synergy may be TNF $\alpha$-dependent in these cell lines.

Birinapant in combination with carboplatin demonstrates efficacy in a subset of primary human ovarian cancers. Molecular alterations that drive OC progression and chemotherapy resistance are highly variable between individual patients. The heterogenous nature of OC tumors poses a challenge in selecting the most effective treatment for individual patients based on their unique tumor traits. This challenge has steered the field of cancer therapeutics towards precision medicine 
A
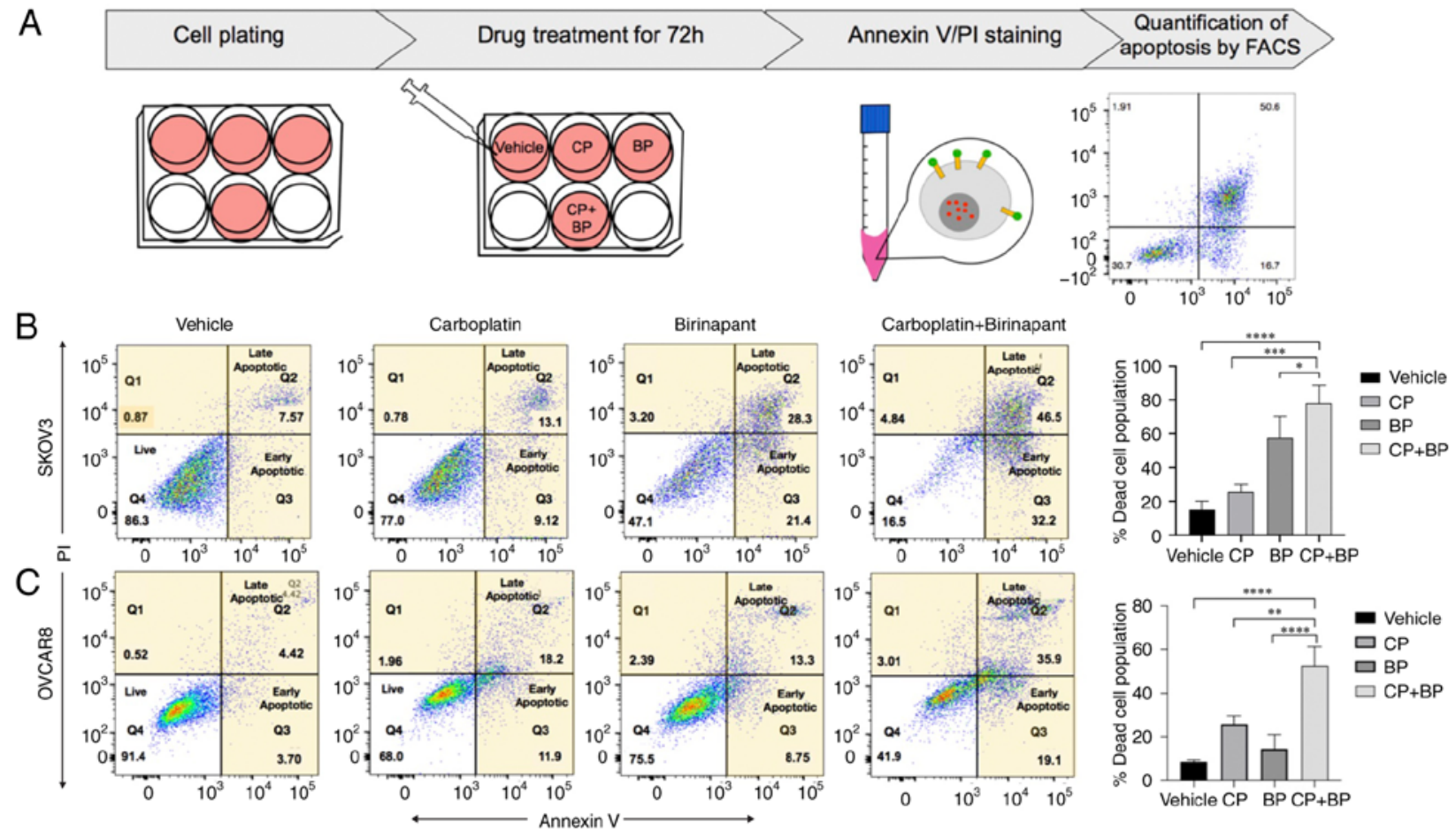

D
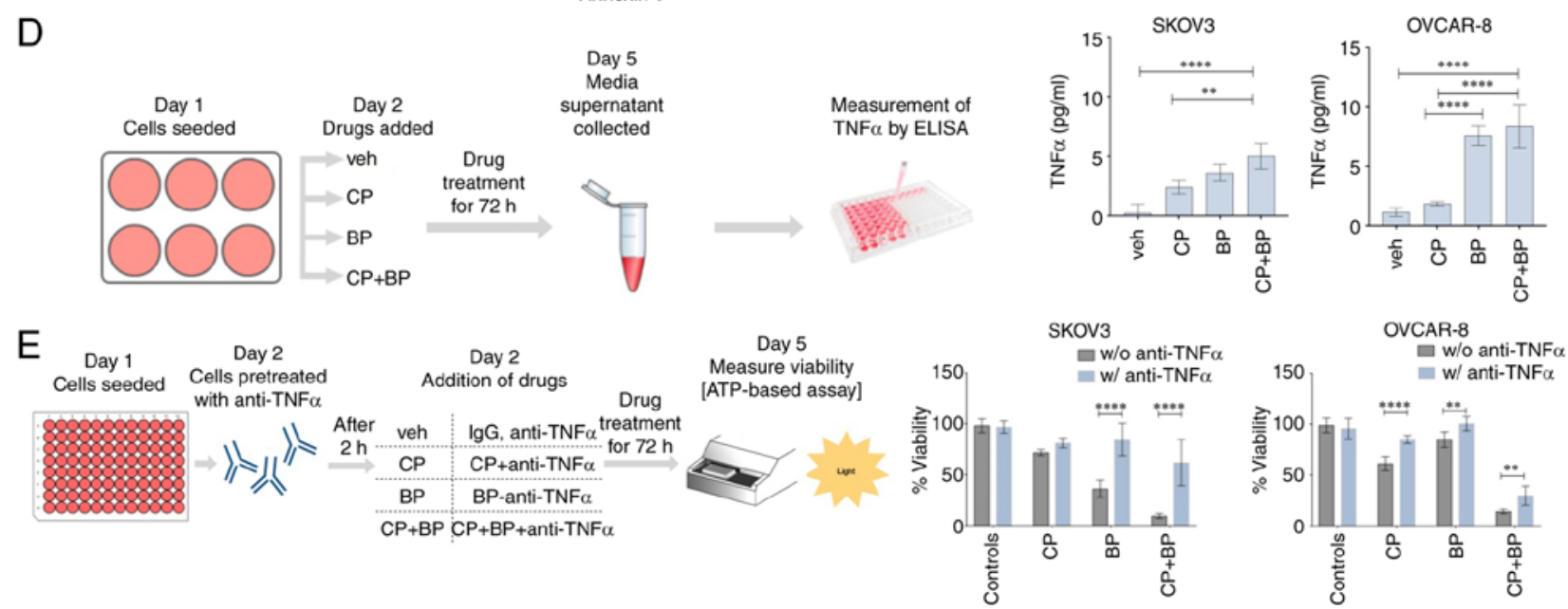

Figure 3. Birinapant and carboplatin combination induces apoptosis in SKOV3 and OVCAR8 cell lines. (A) Experimental schema for measuring drug-induced apoptosis in OVCAR8 and SKOV3 cells using flow cytometry. Cells were treated with carboplatin (CP), birinapant (BP), or co-therapy (CP+BP) for $72 \mathrm{~h}$, stained with FITC-Annexin V and PI, then analyzed by flow cytometry. (B and C) Representative FACS plots showing early apoptotic cells (Q3), late apoptotic cells (Q2), in SKOV3 and OVCAR8 cells (left). Quantification of cell death (right) is measured as \% Annexin $\mathrm{V}^{+}$cell population (Q3+Q2) (mean \pm SEM, $\mathrm{n}=3$, one way-ANOVA, ${ }^{*} \mathrm{P}=0.0248,{ }^{* * *} \mathrm{P}=0.0004,{ }^{* * * *} \mathrm{P}=0.0001$ and ${ }^{* * * * *} \mathrm{P}<0.0001$ ). (D) Workflow for measurement of secreted TNF $\alpha$ using ELISA. SKOV3 and OVCAR8 cells were treated with vehicle (veh), carboplatin (CP), birinapant (BP), or the combination (CP+BP) for $72 \mathrm{~h}$ (left). Quantification of TNFa protein concentration in $\mathrm{pg} / \mathrm{ml}$ for each cell line (right) is shown (mean $\pm \mathrm{SEM}, \mathrm{n}=2$, one-way ANOVA, ${ }^{* * *} \mathrm{P}=0.0032,{ }^{* * * * *} \mathrm{P}<0.0001$ ). (E) Workflow to measure cell death after neutralization with the anti-TNF $\alpha$ antibody. OVCAR8 and SKOV3 cells were pre-treated with anti-TNF $\alpha$ antibody for $2 \mathrm{~h}$ followed by drug treatment for $72 \mathrm{~h}$ (left). Cell viability as measured using the ATP-based luminescence assay is shown (right, mean $\pm \mathrm{SEM}, \mathrm{n}=2$, two-way ANOVA, ${ }^{* *} \mathrm{P}=0.0024$, $\left.{ }^{* * * * *} \mathrm{P}<0.0001\right)$. For all experiments, SKOV3 and OVCAR8 cells were treated with drugs at a concentration corresponding to half maximal inhibitory concentration $\left(\mathrm{IC}_{50}\right.$ ) values [For SKOV3, $(\mathrm{CP})=40 \mu \mathrm{m},(\mathrm{BP})=30 \mathrm{nM}$; for OVCAR8, $\left.(\mathrm{CP})=100 \mu \mathrm{M},(\mathrm{BP})=100 \mathrm{nM}\right]$.

approaches whereby the selection of therapy is tailored to each individual patient's tumor. Exploring the potential of this approach, we utilized the 3D organoid bioassay as a precision medicine tool to test the therapeutic efficacy of carboplatin and birinapant combination in a panel of platinum-resistant primary OC tumor samples (Fig. 4A). A total of 10 platinum-resistant EOC specimens were tested in this study (Fig. 4B).

In this bioassay, dissociated tumor cells either freshly processed or cryopreserved were mixed with Matrigel and plated in tissue culture plates similar to experiments performed using the OC cell lines. Patient-derived organoids were then treated with carboplatin $(0-50 \mu \mathrm{M})$, birinapant $(0-50 \mathrm{nM})$, or co-therapy for 3 consecutive days followed by assessment of cell viability and synergy score calculation (Fig. S3A). Loewe synergy scores calculated by the SynergyFinder tool demonstrated that co-therapy treatment was likely synergistic in targeting $1 / 10$, additive in $7 / 10$, and antagonistic in $2 / 10$ platinum resistant primary tumor samples (Fig. 4B and C). The $\mathrm{IC}_{50}$ of carboplatin and birinapant was also measured for each sample (Fig. S3B). Clinically classified platinum-resistant 
A

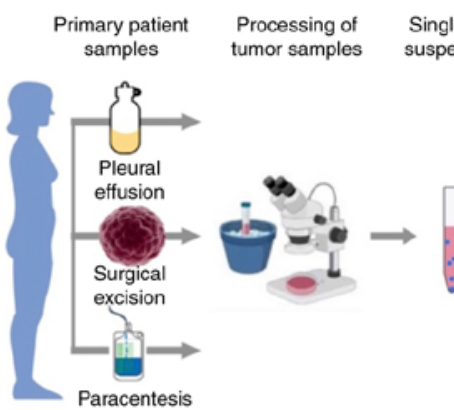

B

\begin{tabular}{|c|c|c|c|}
\hline $\begin{array}{c}\text { Sample } \\
\text { ID }\end{array}$ & Clinical outcome & Synergy score & $\begin{array}{c}\text { Type of drug } \\
\text { interaction }\end{array}$ \\
\hline EOC1 & Platinum refractory & $6.798 \pm 5.84$ & Additive \\
\hline EOC2 & Platinum resistant & $11.377 \pm 2.47$ & Synergistic \\
\hline EOC3 & Platinum resistant & $6.365 \pm 5.66$ & Additive \\
\hline EOC4 & Platinum resistant & $-1.03 \pm 4.07$ & Additive \\
\hline EOC5 & Platinum resistant & $-0.018 \pm 6.84$ & Additive \\
\hline EOC6 & Platinum resistant & $-2.607 \pm 4.01$ & Additive \\
\hline EOC7 & Platinum resistant & $-2.265 \pm 2.68$ & Additive \\
\hline EOC8 & Platinum resistant & $-9.855 \pm 4.81$ & Additive \\
\hline EOC9 & Platinum resistant & $-10.803 \pm 2.08$ & Antagonistic \\
\hline EOC10 & Platinum resistant & $-23.588 \pm 5.3$ & Antagonistic \\
\hline
\end{tabular}

Day 0:

Seeding with

matrigel

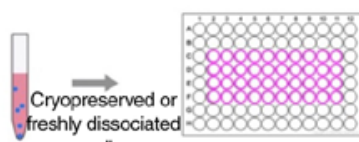

cells

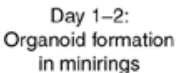

in minirings

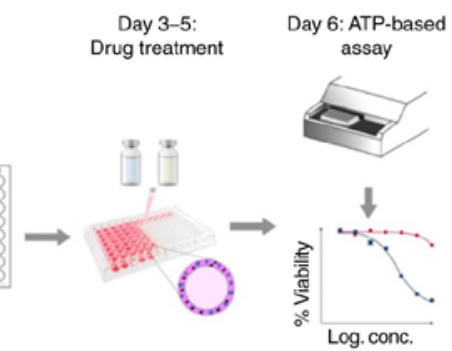

C

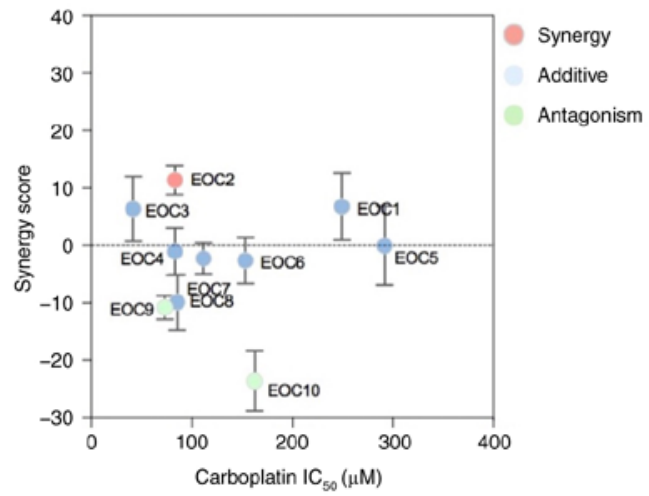

D

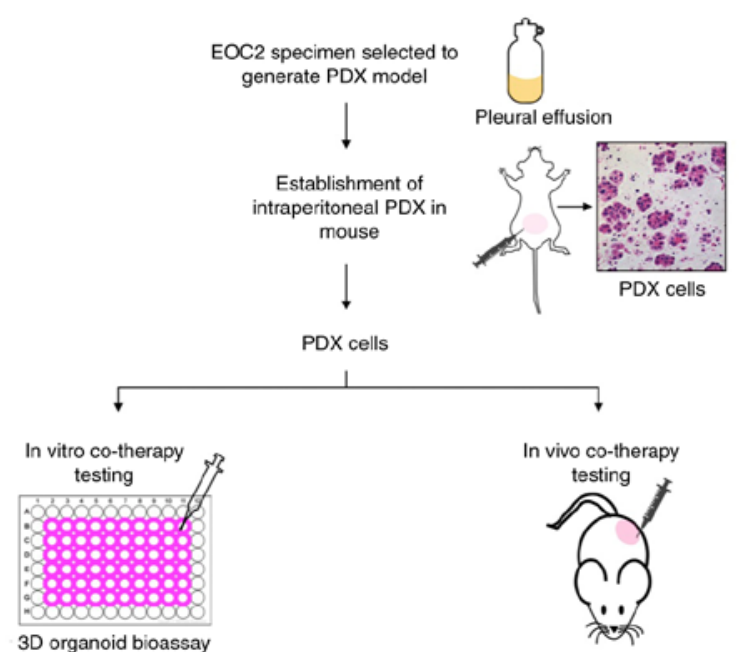

E

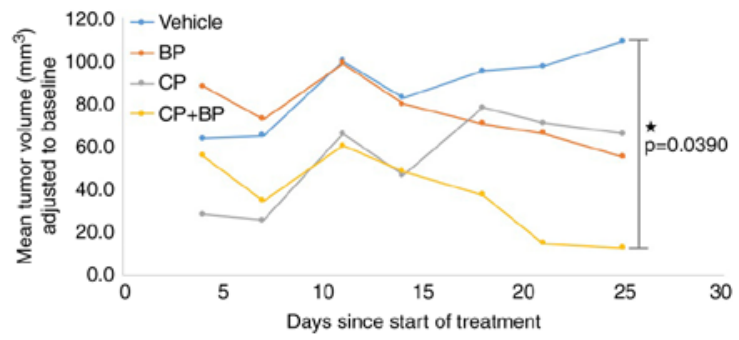

$\mathrm{F}$

$\left.\begin{array}{|c|c|c|}\hline \text { Treatment group } & \begin{array}{c}\text { Mean growth rate } \\ \left(\mathrm{mm}^{3} / \mathrm{days}\right)\end{array} & \text { SEM } \\ \hline \text { Vehicle } & 2.03 & 1.58 \\ \hline \text { Carboplatin } & 2.22 & 0.30 \\ \hline \text { Birinapant } & -1.42 & 0.23 \\ \hline \text { Co-therapy } & -1.94 & 0.59 \\ \hline\end{array}\right]$ *

Figure 4. Testing efficacy of birinapant and carboplatin in targeting platinum-resistant primary patient tumor samples and a PDX model. (A) Workflow to test primary ovarian cancer tumor cells with the carboplatin and birinapant combination using the 3D organoid bioassay. Dissociated primary tumor specimens were grown as organoids and treated with increasing concentrations of carboplatin $(0-50 \mu \mathrm{M})$ and birinapant $(0-50 \mathrm{nM})$ for 3 consecutive days. After $72 \mathrm{~h}$ of drug treatment, cell viability was assessed using an ATP-based luminescence assay. (B) A panel of 10 platinum-resistant epithelial ovarian cancer tumor specimens were utilized to test the in vitro efficacy of co-therapy. Type of drug interaction for each primary tumor specimen is represented as likely synergistic (red), additive (blue), and antagonistic (green). Data represented as synergy score $\pm 95 \%$ confidence interval from independent experiments plated by separate investigators. (C) Calculated drug synergy scores were potted against corresponding carboplatin half maximal inhibitory concentration (IC ( $_{50}$ ) values for each sample. (D) Schema of experimental workflow for generation of a platinum-resistant PDX mouse model, and testing efficacy of co-therapy in vitro and in vivo. (E) Change in tumor volume during the treatment phase. Co-therapy treated mice demonstrated lower tumor burden compared to vehicle after 25 days of treat-

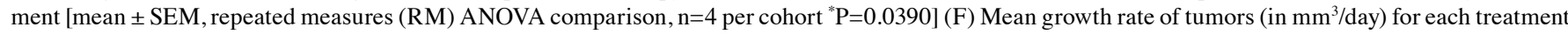
group computed via linear regression model. P-values comparing the mean tumor growth rates are also shown $\left({ }^{* *} \mathrm{P}<0.0001,{ }^{*} \mathrm{P}=0.0365\right)$. Tumor growth rate in birinapant-treated and co-therapy treated mice decreased with time.

tumor samples tested in this bioassay demonstrated high $\mathrm{IC}_{50}$ values of carboplatin (above $40 \mu \mathrm{M}$ ).

To examine the therapeutic efficacy of the co-therapy in vivo, one primary tumor sample that demonstrated maximum
Loewe synergy score in vitro (EOC2) was used to generate a platinum-resistant patient-derived xenograft (PDX) model in immunocompromised mice (Figs. 4D and S4A). The efficacy of the co-therapy was confirmed ex vivo in the $3 \mathrm{D}$ organoid 
bioassay. Similar to its parental patient tumor sample (EOC2), PDX cells were found sensitive to co-therapy (Fig. S4B). Levels of IAP proteins in PDX cells were also measured using western blot analysis (Fig. S4C).

To assess the efficacy of birinapant, carboplatin, and the co-therapy in vivo, PDXs were generated in $\mathrm{n}=17$ female NSG mice by subcuticular injection of one million PDX cells/mouse (Fig. S4D). One mouse was randomly selected and euthanized before treatment to confirm establishment of subcuticular tumors (Fig. S4E). The remaining mice were then randomized into treatment groups ( $\mathrm{n}=4$ /group). Treatment was initiated when the average tumor volume of all PDX-bearing mice reached approximately $125 \mathrm{~mm}^{3}$. Mice were treated with either vehicle, carboplatin $(50 \mathrm{mg} / \mathrm{kg}$ i.p. $1 \mathrm{x} / \mathrm{week})$, birinapant $(30 \mathrm{mg} / \mathrm{kg}$ i.p. $2 \mathrm{x} / \mathrm{wee})$, or the combination of both drugs for 4 weeks. In the co-therapy-treated mice, birinapant was administered $4 \mathrm{~h}$ before carboplatin. During the treatment period, the mouse body weight and size of tumors were recorded twice a week. After 4 weeks of drug treatment, mice were euthanized, and tumors were harvested.

Tumor volume of each mouse was adjusted by subtracting the baseline tumor volume as measured at the start of treatment (day 0 ). At the end of treatment (day 25), the smallest mean tumor volume (adjusted to baseline) was observed in the co-therapy-treated mice compared to vehicle, carboplatin-treated or birinapant-treated group, with mean difference statistically significant with the vehicle-treated mice only ( $\mathrm{P}=0.0390)$ (Fig. 4E).

Comparison of the mean tumor growth rates between treatment groups demonstrated that the mean tumor volume in the vehicle and carboplatin-treated groups increased with time at the rate of $\left(2.03 \pm 1.58 \mathrm{~mm}^{3} /\right.$ day $)$ and $\left(2.22 \pm 0.30 \mathrm{~mm}^{3} / \mathrm{day}\right)$ respectively whereas it decreased in the birinapant-treated and co-therapy-treated mice at the rate of $\left(-1.42 \pm 0.23 \mathrm{~mm}^{3} /\right.$ day $)$ and $\left(-1.94 \pm 0.59 \mathrm{~mm}^{3} /\right.$ day) respectively (Fig. 4F). Tumors harvested after treatment were also weighed and histologically analyzed (Fig. S4F and S4G). IHC staining for the cell proliferation biomarker Ki-67 in these tumor fragments demonstrated no significant differences in the percentage of Ki67-positive cells across the 4 treatment groups (Fig. S4H). The mean number of mitosis (per high power field) was found lower in the birinapant (0.6377) and co-therapy (0.720)-treated groups compared to the vehicle (1.533) and carboplatin (1.388) groups, although it did not reach statistical significance (Fig. S4I). No signs of drug toxicity as measured by mouse body weight were observed (Fig. S4J).

Overall, the results highlight consistency between in vitro and in vivo therapeutic responses of cancer cells to combination therapy as measured by the 3D organoid bioassay. This suggests that the 3D organoid bioassay may be used as valuable preclinical research tool in the field of cancer therapeutics to evaluate the efficacy of targeted therapies. Additionally, the results indicate the efficacy of carboplatin and birinapant combination in targeting a subset of platinum-resistant primary human ovarian cancers.

\section{Discussion}

In the present study, we utilized a 3D-organoid bioassay $(28,29)$ as an in vitro platform to measure platinum sensitivity of ovarian cancer cell lines. Assay-predicted results were found to be correlated with reported platinum sensitivities for each cell line. The bioassay was also used to test platinum sensitivity of
10 primary patient tumor samples. We observed that clinically classified platinum-resistant tumor samples demonstrated high $\mathrm{IC}_{50}$ values for carboplatin that ranged from 40 to $291.8 \mu \mathrm{M}$. These results suggest that the 3D-organoid bioassay may be utilized as a potential companion diagnostic $(\mathrm{CDx})$ for predicting ovarian cancer patients' response to platinum-based chemotherapy. As a potential precision medicine tool, this bioassay offers several advantages $(28,29)$. First, as demonstrated in this study, the bioassay allows formation of epithelial ovarian cancer organoids from fluid samples (ascites, pleural effusions) as well as from dissociated tumor cells obtained from surgical specimens. Although no biopsy samples were tested in this study, the low cellular requirement would make this assay compatible for testing small biopsies as well. Second, assay results were obtained within one week, as previously reported (28), making it suitable for time-sensitive therapeutic decision making. However, for clinical utility, the bioassay needs to be validated prospectively with a large cohort of ovarian cancer tumor samples in a clinical trial.

Another major goal of this study was to target platinum-resistant epithelial ovarian cancer cells. Chemo-resistant cancer cells are known to evade therapy-induced apoptosis. As a promising therapeutic strategy for activating apoptosis in ovarian cancer cells, we utilized a small molecule inhibitor called birinapant designed to inhibit anti-apoptotic IAP proteins in combination with carboplatin. The in vitro efficacy of the co-therapy was tested across a panel of epithelial ovarian cancer cell lines in the 3D organoid bioassay. Results from these studies demonstrated enhanced cell death for a subset of platinum-resistant cell lines treated with the co-therapy compared to single agents. Similarly, the combination of birinapant and carboplatin was found effective in targeting a subset of platinum-resistant primary patient tumor samples in vitro. The combination therapy also demonstrated some efficacy in targeting a platinum-resistant PDX model. The correlation between in vitro and in vivo therapeutic response of these PDX cells and the parental human tumor demonstrates the potential of the 3D organoid bioassay as a high throughput drug testing platform.

Our findings in the present study are consistent with published data demonstrating that combining SMAC mimetics with carboplatin could target ovarian cancers using in vitro and in vivo preclinical models $(26,40)$. Several studies have explored birinapant activity in combination with different anticancer agents. The combination of birinapant with several chemotherapies including carboplatin/paclitaxel, docetaxel, irinotecan, gemcitabine or liposomal doxorubicin have also been evaluated in a Phase I/II clinical trial for treatment of patients with advanced or metastatic solid tumors (NCT01188499). The results from this clinical trial demonstrated that birinapant could be well-tolerated when combined with multiple chemotherapies (44). Birinapant has also been tested in combination with gemcitabine, oxaliplatin/5-fluorouracil, TNF $\alpha$, TRAIL and docetaxel for targeting preclinical models of breast cancer (24), colorectal cancer (45), and head and neck squamous cell carcinomas (HNSCC) respectively (46). Other than standard chemotherapy drugs, birinapant has also shown synergy with CAR-T therapy and radiotherapy in targeting colorectal cancer (47) and HNSCC (22), respectively. A recent study has suggested the significance of sequential drug administration for increased drug synergy between birinapant and carboplatin in targeting OVCAR8 xenografts (26). Here, birinapant 
was injected after carboplatin administration. In our study, we administered birinapant $4 \mathrm{~h}$ prior to carboplatin injection in the PDX-bearing mice. The rationale for this sequence of drug administration was to activate birinapant-mediated apoptosis in the tumor cells to better prime them for carboplatin-induced cytotoxicity. The optimal sequence for administration of these two drugs may require more investigation.

Although the development of birinapant as an anticancer therapeutic is promising, there remain some challenges to be addressed. One of the main challenges is to identify and develop biomarkers of response to birinapant. Several studies have assessed and demonstrated that IAP levels do not correlate with sensitivity to birinapant $(48,49)$. Zinngrebe et al demonstrated that protein expression levels of cIAP1 and XIAP were similar in SMAC mimetic-sensitive and SMAC mimetic-insensitive primary B-cell acute lymphoblastic leukemia samples (48). McCann et al also reported that expression of IAP proteins alone could not be correlated to birinapant sensitivity in a panel of colorectal cancer cell lines (49). Similarly, in our study, we did not see a correlation between IAP protein levels and response to birinapant in the ovarian cancer cell lines tested. In an attempt to characterize predictive biomarkers of response to birinapant, a recent study has identified a 12-protein signature consisting of apoptotic proteins that could segregate responders and non-responders to birinapant and chemotherapy combinations in colorectal cancer (49). Another study identified a biomarker set of 4 genes including TNFRSF1A, TSPAN7, DIPK1C and $M T X 2$ for prediction of response to SMAC mimetics in pediatric precursor-cell acute lymphoblastic leukemia (48). Although these studies have paved the way for personalized treatment of cancer using SMAC mimetics, their successful clinical implementation across different cancers requires further evaluation in clinical trials. In the absence of any reliable biomarkers, optimization of the 3D organoid bioassay may potentially offer a precision medicine tool to predict therapeutic responses ex vivo.

Despite these shortcomings, the antitumor activity and safety profile of birinapant highlights its potential as an effective anticancer drug when combined with other chemotherapeutics. In summary, work by other investigators and results from this study provide a rationale for evaluation of the efficacy of birinapant in combination with carboplatin in clinical trials for patients with platinum-resistant ovarian cancers.

\section{Acknowledgements}

We would like to thank Dr Jeffrey Gornbein (biostatistician), the UCLA Translational Pathology Core Laboratory (TPCL) and the Broad Stem Cell Research Center (BSCRC) Flow Cytometry Core for their assistance. We also thank the patients and their relatives without whom this study would not have been possible.

\section{Funding}

AN and SM are partially supported by a Department of Veterans Affairs Merit Award (grant no. I01BX004651).

\section{Availability of data and materials}

All data generated or analyzed during the current study are included in this article. Data sharing is not applicable to this article, as no datasets were generated or analyzed during the current study.

\section{Authors' contributions}

Conception and design were carried out by TS and SM. In vitro assays were conducted by $\mathrm{TS}$ and $\mathrm{AN}$. Processing of patient tumor samples was accomplished by AN. The animal study was performed by TS, AN and NR. The IHC study was conducted by GD and NAM. Writing, review of the manuscript was conducted by TS, AN, and SM. Study supervision was carried out by TS and SM. All authors read and approved the manuscript and confirmed the generated data and agree to be accountable for all aspects of the research in ensuring that the accuracy or integrity of any part of the work are appropriately investigated and resolved.

\section{Ethics approval and consent to participate}

All primary patient tumor samples tested in this study were obtained from consented patients through protocols approved by the UCLA Office of the Human Research Protection Program (IRB\# 10-000727). All animal experiments were approved by the UCLA Animal Research Committee (protocol 2008-153) and conducted under the supervision of the UCLA Division of Laboratory Animal Medicine.

\section{Patient consent for publication}

Patients provided written informed consent for publication of any associated data maintaining their identity confidentiality.

\section{Competing interests}

Authors declare no competing interests.

\section{References}

1. Siegel RL, Miller KD, Fuchs HE and Jemal A: Cancer Statistics, 2021. CA Cancer J Clin 71: 7-33, 2021.

2. Desai A, Xu J, Aysola K, Qin Y, Okoli C, Hariprasad R, Chinemerem U, Gates C, Reddy A, Danner O, et al: Epithelial ovarian cancer: An overview. World J Transl Med 3: 1-8, 2014.

3. Kurnit KC, Fleming GF and Lengyel E: Updates and new options in advanced epithelial ovarian cancer treatment. Obstet Gynecol 137: 108-121, 2021.

4. Kaplan DA: Overview of the Updated NCCN Guidelines on Ovarian Cancer. 6, 2020.

5. Berek JS, Crum C and Friedlander M: Cancer of the ovary, fallopian tube, and peritoneum. Int J Gynaecol Obstet 119 (Suppl 2): S118-S129, 2012.

6. Baert T, Ferrero A, Sehouli J, O'Donnell DM, González-Martín A, Joly F, van der Velden J, Blecharz P, Tan DSP, Querleu D, et al: The systemic treatment of recurrent ovarian cancer revisited. Ann Oncol 32: 710-725, 2021.

7. Yang Y, Yang Y, Yang J, Zhao X and Wei X: Tumor microenvironment in ovarian cancer: Function and therapeutic strategy. Front Cell Dev Biol 8: 758, 2020.

8. Zhou J, Kang Y, Chen L, Wang H, Liu J, Zeng S and Yu L: The drug-resistance mechanisms of five platinum-based antitumor agents. Front Pharmacol 11: 343, 2020.

9. Dubrez L, Berthelet J and Glorian V: IAP proteins as targets for drug development in oncology. Onco Targets Ther 9: 1285-1304, 2013.

10. Finlay D, Teriete P, Vamos M, Cosford NDP and Vuori K: Inducing death in tumor cells: Roles of the inhibitor of apoptosis proteins. F1000Res 6: 587, 2017. 
11. Pluta P, Jeziorski A, Cebula-Obrzut AP, Wierzbowska A Piekarski J and Smolewski P: Expression of IAP family proteins and its clinical importance in breast cancer patients. Neoplasma 62: 666-673, 2015

12. Hofmann HS, Simm A, Hammer A, Silber RE and Bartling B: Expression of inhibitors of apoptosis (IAP) proteins in non-small cell human lung cancer. J Cancer Res Clin Oncol 128: 554-560, 2002.

13. Imoto I, Tsuda H, Hirasawa A, Miura M, Sakamoto M, Hirohashi S and Inazawa J: Expression of cIAP1, a target for 11q22 amplification, correlates with resistance of cervical cancers to radiotherapy. Cancer Res 62: 4860-4866, 2002.

14. Miyamoto M, Takano M, Iwaya K, Shinomiya N, Kato M, Aoyama T, Sasaki N, Goto T, Suzuki A, Hitrata J and Furuya K: $\mathrm{X}$-chromosome-linked inhibitor of apoptosis as a key factor for chemoresistance in clear cell carcinoma of the ovary. $\mathrm{Br}$ J Cancer 110: 2881-2886, 2014

15. Cai Y, Ma W, Huang X, Cao L, Li H, Jiang Y, Lu N and Yin Y: Effect of survivin on tumor growth of colorectal cancer in vivo. Int J Clin Exp Pathol 8: 13267-13272, 2015.

16. Zhao G, Wang Q, Wu Z, Tian X, Yan H, Wang B, Dong P, Watari H, Pfeffer LM, Guo Y, et al: Ovarian primary and metastatic tumors suppressed by survivin knockout or a novel survivin inhibitor. Mol Cancer Ther 18: 2233-2245, 2019.

17. Park E, Gang EJ, Hsieh YT, Schaefer P, Chae S, Klemm L, Huantes S, Loh M, Conway EM, Kang ES, et al: Targeting survivin overcomes drug resistance in acute lymphoblastic leukemia. Blood 118: 2191-2199, 2011.

18. Moriai R, Tsuji N, Moriai M, Kobayashi D and Watanabe N: Survivin plays as a resistant factor against tamoxifen-induced apoptosis in human breast cancer cells. Breast Cancer Res Treat 117: 261-271, 2009

19. Span PN, Sweep FCGJ, Wiegerinck ET, Tjan-Heijnen VC, Manders P, Beex LV and de Kok JB: Survivin is an independent prognostic marker for risk stratification of breast cancer patients. Clin Chem 50: 1986-1993, 2004.

20. Sui L, Dong Y, Ohno M, Watanabe Y, Sugimoto K and Tokuda M: Survivin expression and its correlation with cell proliferation and prognosis in epithelial ovarian tumors. Int J Oncol 21: 315-320, 2002.

21. Morrish E, Brumatti G and Silke J: Future therapeutic directions for Smac-Mimetics. Cells 9: 406, 2020.

22. Eytan DF, Snow GE, Carlson S, Derakhshan A, Saleh A, Schiltz S, Cheng H, Mohan S, Cornelius S, Coupar J, et al: SMAC mimetic birinapant plus radiation eradicates human head and neck cancers with genomic amplifications of cell death genes FADD and BIRC2. Cancer Res 76: 5442-5454, 2016.

23. Lalaoui N, Merino D, Giner G, Vaillant F, Chau D, Liu L, Kratina T, Pal B, Whittle JR, Etemadi N, et al: Targeting triple-negative breast cancers with the Smac-mimetic birinapant Cell Death Differ 27: 2768-2780, 2020

24. Xie X, Lee J, Liu H, Pearson T, Lu AY, Tripathy D, Devi GR, Bartholomeusz C and Ueno NT: Birinapant enhances gemcitabine's antitumor efficacy in triple-negative breast cancer by inducing intrinsic pathway-dependent apoptosis. Mol Cancer Ther 20: 296-306, 2021 .

25. Colombo M, Marabese M, Vargiu G, Broggini M and Caiola E: Activity of birinapant, a SMAC mimetic compound, alone or in combination in NSCLCs with different mutations. Front Oncol 10: 532292, 2020.

26. Hernandez LF, Dull AB, Korrapati S and Annunziata CM: Smac-mimetic enhances antitumor effect of standard chemotherapy in ovarian cancer models via Caspase 8-independent mechanism. Cell Death Discov 7: 134,2021.

27. Noonan AM, Bunch KP, Chen JQ, Herrmann MA, Lee JM, Kohn EC, O'Sullivan CC, Jordan E, Houston N, Takebe N, et al: Pharmacodynamic markers and clinical results from the phase II Study of the SMAC-Mimetic birinapant in women with relapsed platinum-resistant or refractory epithelial ovarian cancer. Cancer 122: 588-597, 2016.

28. Phan N, Hong JJ, Tofig B, Mapua M, Elashoff D, Moatamed NA, Huang J, Memarzadeh S, Damoiseaux R and Soragni A: A simple high-throughput approach identifies actionable drug sensitivities in patient-derived tumor organoids. Commun Biol 2: 78, 2019.

29. Nguyen HTL and Soragni A: Patient-derived tumor organoid rings for histologic characterization and high-throughput screening. STAR Protoc 1: 100056, 2020

30. Ianevski A, Giri AK and Aittokallio T: SynergyFinder 2.0: Visual analytics of multi-drug combination synergies. Nucleic Acids Res 48(W1): W488-W493, 2020.

31. National Cancer Institute: Oxaliplatin. Accessed September 15, 2021. Available from: https://www.cancer gov/about-cancer/treatment/drugs/oxaliplatin.
32. National Cancer Institute: Cisplatin. Accessed September 15 , 2021. Available from: https://www.cancer.gov/about-cancer/treatment/drugs/cisplatin.

33. National Cancer Institute: Discovery-Cisplatin and The Treatment of Testicular and Other Cancers. Accessed September 15 2021. Available from: https://www.cancer.gov/research/progress/discovery/cisplatin.

34. Decatris MP, Sundar S and O'Byrne KJ: Platinum-based chemotherapy in metastatic breast cancer: Current status. Cancer Treat Rev 30: 53-81, 2004

35. Burstein HJ, Mangu PB, Somerfield MR, Schrag D, Samson D, Holt L, Zelman D and Ajani JA; American Society of Clinical Oncology: American Society of Clinical Oncology clinical practice guideline update on the use of chemotherapy sensitivity and resistance assays. J Clin Oncol 29: 3328-3330, 2011.

36. Haley J, Tomar S, Pulliam N, Xiong S, Perkins SM, Karpf AR, Mitra S, Nephew KP and Mitra AK: Functional characterization of a panel of high-grade serous ovarian cancer cell lines as representative experimental models of the disease. Oncotarget 7 32810-32820, 2016.

37. Beaufort CM, Helmijr JC, Piskorz AM, Hoogstraat M, Ruigrok-Ritstier K, Besselink N, Murtaza M, van IJcken WF, Heine AA, Smid M, et al: Ovarian cancer cell line panel (OCCP): Clinical importance of in vitro morphological subtypes. PLoS One 9: e103988, 2014

38. Kopper O, de Witte CJ, Lõhmussaar K, Valle-Inclan JE, Hami N, Kester L, Balgobind AV, Korving J, Proost N, Begthel H, et al: An organoid platform for ovarian cancer captures intra- and interpatient heterogeneity. Nat Med 25: 838-849, 2019.

39. LaCasse EC, Mahoney DJ, Cheung HH, Plenchette S, Baird S and Korneluk RG: IAP-targeted therapies for cancer. Oncogene 27: 6252-6275, 2008.

40. Thibault B, Genre L, Le Naour A, Broca C, Mery E, Vuagniaux G, Delord JP, Wiedemann N and Couderc B: DEBIO 1143, an IAP inhibitor, reverses carboplatin resistance in ovarian cancer cells and triggers apoptotic or necroptotic cell death. Sci Rep 8: 17862, 2018.

41. Rabik CA and Dolan ME: Molecular mechanisms of resistance and toxicity associated with platinating agents. Cancer Treat Rev 33: 9-23, 2007.

42. Vince JE, Wong WW, Khan N, Feltham R, Chau D, Ahmed AU, Benetatos CA, Chunduru SK, Condon SM, McKinlay M, et al: IAP Antagonists Target cIAP1 to Induce TNF $\alpha$-Dependent Apoptosis. Cell 131: 682-693, 2007.

43. Probst BL, Liu L, Ramesh V, Li L, Sun H, Minna JD and Wang L: Smac mimetics increase cancer cell response to chemotherapeutics in a TNF- $\alpha$-dependent manner. Cell Death Differ 17: 1645-1654, 2010.

44. Amaravadi RK, Senzer NN, Martin LP, Schilde RJ, LoRusso P, Papadopoulos KP, Weng DE, Graham M and Adjei AA: A phase I study of birinapant (TL32711) combined with multiple chemotherapies evaluating tolerability and clinical activity for solid tumor patients. J Clin Oncol 31 (Suppl 15): S2504, 2013.

45. Fichtner M, Bozkurt E, Salvucci M, McCann C, McAllister KA, Halang L, Düssmann H, Kinsella S, Crawford N, Sessler T, et al: Molecular subtype-specific responses of colon cancer cells to the SMAC mimetic Birinapant. Cell Death Dis 11: 1020, 2020.

46. Eytan DF, Snow GE, Carlson SG, Schiltz S, Chen Z and Van Waes C: Combination effects of SMAC mimetic birinapant with TNF $\alpha$, TRAIL, and docetaxel in preclinical models of HNSCC. Laryngoscope 125: E118-E124, 2015.

47. Michie J, Beavis PA, Freeman AJ, Vervoort SJ, Ramsbottom KM, Narasimhan V, Lelliott EJ, Lalaoui N, Ramsay RG, Johnstone RW, et al: Antagonism of IAPs Enhances CAR T-cell Efficacy. Cancer Immunol Res 7: 183-192, 2019.

48. Zinngrebe J, Schlichtig F, Kraus JM, Meyer M, Boldrin E, Kestler HA, Meyer LH, Fischer-Posovszky P and Debatin KM: Biomarker profile for prediction of response to SMAC mimetic monotherapy in pediatric precursor B-cell acute lymphoblastic leukemia. Int J Cancer 146: 3219-3231, 2020.

49. McCann C, Matveeva A, McAllister K, Van Schaeybroeck S, Sessler T, Fichtner M, Carberry S, Rehm M, Prehn JHM and Longley DB: Development of a protein signature to enable clinical positioning of IAP inhibitors in colorectal cancer. FEBS J 288: 5374-5388, 2021.

This work is licensed under a Creative Commons Attribution-NonCommercial-NoDerivatives 4.0 International (CC BY-NC-ND 4.0) License. 